A puntes del CEN ES

ISSN 0120-3053

Volumen 31 - №. 53

Primer Semestre 2012

Págs. 179-199

\title{
Estimación y análisis de los gastos e ingresos económicos de la población flotante estudiantil universitaria de pregrado en la ciudad de Tunja, Colombia
}

Estimation and analysis of expenditure and income of the floating population of undergraduate university student in the city of Tunja, Colombia

María Isabel Rojas Triana*

Fecha de recepción: 25 de agosto de 2011

Fecha de aprobación: 24 de noviembre de 2011

Licenciada en Matemáticas y Estadística. Especialista en Estadística Aplicada. Magíster en Ciencias Económicas. Docente Catedrática Escuela de Matemáticas y Estadística, Universidad Pedagógica y Tecnológica de Colombia, sede Tunja. Correo electrónico: marisaestadistica@hotmail.com 


\title{
Resumen
}

Este artículo muestra la estimación de un modelo de demanda del gasto e ingreso económico que ejercen los estudiantes flotantes en la ciudad de Tunja. Está basado en una aplicación a la función de Engel propuesta por Working-Lesser, tomando datos de una encuesta hecha a una muestra de estudiantes flotantes universitarios de pregrado de la ciudad de Tunja, a quienes se les indaga por algunas variables socioeconómicas, entre ellas el gasto y el ingreso. La demanda más relevante de los estudiantes flotantes está dada por el gasto que ejercen en vivienda, observado a partir del valor de los coeficientes del sistema de ecuaciones planteado en el modelo.

Palabras clave: población migrante temporal, impacto sobre consumo, comportamiento del gasto e ingreso, aplicación de la curva de Engel, M étodo de Working y Lesser.

Clasificación JEL: J62, 123, D12, D31, C39

\begin{abstract}
This article shows the estimated demand model of expenditure and income of floating students in the city of Tunja. It is based on an application to the Engel curve, using the method of Working and Leser, taking data from a poll made on a sample of floating university students in the city of Tunja, whom were asked about some socio-economic variables, such as spending and income. The most important demand of floating students is given by spending on housing, which is seen from the value of the coefficients of the equation system proposed in the model.
\end{abstract}

Keywords: temporary migrant population, consumption impact, behavior of expense and income, application of Engel curve, Working and Lesser method.

Jel Classification: J62, 123, D12, D31, C39 


\section{Introducción}

Para muchos autores, la migración en población se define como el tiempo mínimo de un lustro de estadía en un lugar determinado, regresando luego a su lugar de origen. Si la estadía corresponde a un tiempo inferior o igual a un año, Françoise Dureau (2001), la considera como población flotante.

Si relacionamos "población flotante" con "población migrante", las dos nociones implican un traslado de residencia que realizan las personas de una localidad a otra, cruzando fronteras o límites de una región geográfica, diferenciándose en el tiempo de estadía.

En Colombia la magnitud de población migrante se determina mediante registros permanentes en censos y encues- tas especializadas y se construye a partir de información sobre el lugar de residencia actual, lugar de nacimiento y lugar de residencia en algún momento específico anterior. Dureau (2001) argumenta que, tanto en las ciudades como a nivel nacional, el aumento de los sistemas residenciales complejos está basado en estadías cíclicas o temporales; los censos son poco adecuados para dar cuenta de la realidad de estas prácticas espaciales de la población, pues no toman en cuenta sino algunas formas de movilidad espacial, en este caso, se le llama migración definitiva.

La población estimada en los censos diseñados para ello, corresponde al concepto demográfico tradicional de residente habitual, Io cual supone implícitamente que la residencia es permanente y única, considerando 
exclusivamente a las personas que residen habitualmente en las viviendas. Sin embargo, se afirma también, que una cierta proporción de la población no reside en un lugar único, sino en varios, desplazándose continuamente entre ellos, denominándose, así, población flotante, refiriéndose a personas no residentes habituales en viviendas que cumplen la condición de haber permanecido por lo menos 28 días en la vivienda durante el año de referencia (12 meses anteriores a la encuesta).

De otro lado, algunos estudios de Leiwen Jiang (2006), indican que la población con movilidad flotante, se acomoda en forma espontánea, sin la planeación de vivienda eficiente y sin el control de la demanda en algunos bienes y servicios.

Tunja presenta alto porcentaje en población flotante estudiantil universitaria como se evidencia en anteriores estudios (Rojas, 2009), indicadores hipotéticos observan procesos de transformación en el uso del suelo urbano, por ejemplo, un $47 \%$ de los estudiantes tienen en alquiler habitaciones en condiciones de hacinamiento, consecuencia del afán de la oferta en vivienda por cubrir las exigencias del mer- cado, agregado a la alta proliferación de bares y negocios destinados a captar masa estudiantil.

Uno de los aspectos positivos, en el sentido económico, de la población flotante de cualquier lugar, podría serel incremento en la demanda de bienes y servicios, en la medida en que propicie más ingresos a las familias $y$, en consecuencia, los de la ciudad o de la región.

Teniendo en cuenta los anteriores aspectos en población flotante, es importante destacar la participación de las universidades de la ciudad de Tunja, en población estudiantil flotante dada en la tabla 1.

L as cifras muestran que el $\mathbf{6 4} \%$ de los estudiantes matriculados en el II semestre del año 2007 del total de estudiantes en programas de pregrado, son flotantes. Según otros datos encontrados: el $85 \%$ de ellos provienen de todos los municipios del departamento de Boyacá, siendo la mayor parte de Sogamoso y Duitama y el resto (15\%) vienen de otros departamentos de Colombia, tales como Santanderes, Casanare, Tolima, Nariño, M eta, Casanare, Costa Atlántica y Pacífica, entre otros, y de otros países como M éxico y Francia. 
Tabla 1. Población estudiantil universitaria de pregrado matriculada II Semestre de 2007

\begin{tabular}{|l|c|c|c|c|}
\hline Universidad & $\begin{array}{c}\text { Total } \\
\text { matrícula } \\
\text { (2) }\end{array}$ & $\begin{array}{c}\text { No. estudiantes } \\
\text { flotantes } \\
\text { (3) }\end{array}$ & $\begin{array}{c}\text { Porcentaje } \\
\text { flotantes } \\
\text { (3)/13.226 }\end{array}$ & $\begin{array}{c}\text { Total } \\
\text { (\%) } \\
\text { participación } \\
\text { /universidad } \\
\text { (3)/(2) }\end{array}$ \\
\hline UPTC Presencial & 10798 & 6899 & 52 & 64 \\
UPTC Distancia & 1040 & 602 & 5 & 58 \\
Universidad de Boyacá & 5180 & 3172 & 24 & 61 \\
Universidad Santo Tomás & 1683 & 1567 & 12 & 93 \\
Escuela de A dministración & 170 & 70 & 1 & 41 \\
$\begin{array}{l}\text { Pública (ESA P) } \\
\text { Universidad Antonio Nariño }\end{array}$ & 480 & 96 & 1 & 20 \\
$\begin{array}{l}\text { Universidad N acional A bierta } \\
\text { y a Distancia (UN ND) }\end{array}$ & 519 & 415 & 3 & 80 \\
Fundación Universitaria Juan & 810 & 405 & 3 & 50 \\
de Castellanos & 20680 & 13226 & 100 & 64 \\
TOTALES & & & & \\
\hline
\end{tabular}

Fuente: oficinas de planeación y sistemas de cada una de las universidades (total de población estudiantil). Cálculos de participación elaborados por la autora.

Esta población, también Ilamada migrante temporal, no solo aumenta el tamaño de la población local por temporadas, sino que genera una recomposición social espacial urbana, evidenciada en la concentración de población que se ubica en barrios aledaños a las universidades; impulsando de manera simultánea la oferta de servicios en supermercados, restaurantes, sitios de recreación o de diversión (bares), entidades financieras y transporte, tanto urbano como municipal, y prácticas de alojamiento. Cabe entonces preguntarnos ¿en qué medida la creciente población flotante universitaria produce cambios en la demanda de bienes y servicios en la ciudad?
El objetivo de este artículo es hacer un análisis del gasto y su asignación en el consumo de algunos bienes y servicios relevantes de la población flotante estudiantil universitaria de Tunja, aplicando la función de Engel propuesta por Working y Lesser. Con datos obtenidos de una encuesta aplicada a una muestra probabilística de tamaño 847 de una población total de 13.226 estudiantes universitarios de pregrado, de las universidades más representativas en población estudiantil flotante en la ciudad de Tunja. La encuesta se hizo mediante muestreo aleatorio simple, con un nivel de confianza del $95 \%$ y un error del $5 \%$. Los estudiantes fueron caracterizados 
por sus ingresos con varianza homogénea (veáse el promedio de la composición del ingreso sección 2.1) para las universidades en estudio, a quienes se les indagó sobre algunas variables socioeconómicas, entre ellas el ingreso económico y el gasto en el consumo de algunos bienes y servicios que demandan en la ciudad.

Los datos obtenidos son de corte transversal y no incluyen variaciones de precios. En este estudio solo nos centraremos en las variables gasto e ingreso.En la segunda parte se presenta una descripción de la composición, origen y asignación de los ingresos económicos y gastos de los estudiantes flotantes universitarios en estudio.

En la tercera parte se presentan las formas funcionales matemáticas que relacionan el gasto/ingreso sobre algunos servicios que adquiere la población flotante estudiantil de la ciudad de Tunja, mediante una aplicación a la curva de Engel por el método de Working y Leser.

A I modelo de Engel, de la forma funcional estocástica, se implementa un sistema de ecuaciones simultáneas lineales del gasto total respecto al gasto proporcional de algunos bienes de consumo relevante; cada una de ellas corresponde a un modelo de regresión lineal simple (nivel- log) en cada uno de los bienes aplicados al sistema de Working y Lesser.
$L$ as primeras aplicaciones fueron realizadas por Working (1943) y posteriormente por Leser (1963, 1976), Deaton y Muellbauer (1980). En CoIombia y Bolivia por M uñoz (1999) y Jiménez (2000). En estas se analizó el comportamiento del gasto de los hogares colombianos y bolivianos, tomando datos de las encuestas de hogares realizada por el DANE-Colombia y PASI - Bolivia (Programas de A sistencia Social Integral).

En la cuarta parte se hace un análisis y discusión de los resultados de las variables gasto en: alimentación, arriendo, transporte, apoyos académicos (útiles escolares, papelería, fotocopias) de la encuesta aplicada, usando los datos recolectados a la población estudiantil flotante de las universidades más representativas en población flotante en Tunja, del II semestre de 2007, fecha en la cual se hace corte para el estudio. Por último se dan conclusionesy referencias bibliográficas.

\section{Descripción del ingreso y gastos sobre el consumo de algunos bienes y servicios}

\subsection{Composición, origen y asignación de los ingresos económicos de los es- tudiantes flotantes universitarios}

Los estudiantes flotantes obtienen ingresos en mayor promedio provenientes de la familia, y en menor promedio por becas y préstamos del Icetex. 
El promedio de ingresos totales mensuales de un estudiante es de $\$ 545.575,80$ corrientes, mayor a un salario mínimo legal vigente del periodo de encuesta ${ }^{1}$, con una desviación de \$589.591,30; existen estudiantes con ingresos superiores a $\$ 1^{\prime} 200.000$ mientras que otros no registran ingresos (ver Tabla 2. Estadísticas descriptivas de la distribución del ingreso).
Se encontró alta variación en ingresos por concepto de becas, debido a que solo un $2 \%$ del total (835) de estudiantes se benefician de estas. N ótese también, que más de la mitad de estudiantes, equivalente al $52 \%$, tienen ingresos totales menores e iguales a un salario mínimo legal vigente (s.m.l.v.) mensual, Io cual indica que buena parte de estudiantes se mantienen con recursos notablemente bajos. (ver tabla 3).

Tabla 2. Estadísticas descriptivas distribución de ingresos promedios mensuales (pesos corrientes)

\begin{tabular}{|l|c|c|c|c|c|}
\hline $\begin{array}{c}\text { Estadísticos } \\
\text { descriptivos / } \\
\text { Orígen de } \\
\text { los Ingresos }\end{array}$ & $\begin{array}{c}\text { Número de } \\
\text { Estudiantes } \\
\text { Beneficiados }\end{array}$ & $\begin{array}{c}\text { Promedio } \\
\text { (Pesos } \\
\text { corrientes) }\end{array}$ & $\begin{array}{c}\text { Desviación } \\
\text { Típica (Pesos } \\
\text { Corrientes) }\end{array}$ & $\begin{array}{c}\text { Coeficiente } \\
\text { de } \\
\text { Variación }\end{array}$ & $\begin{array}{c}\text { Porcentaje } \\
\text { Estudiantes } \\
\text { Beneficiados }\end{array}$ \\
\hline Familia & & 651 & $360.173,17$ & $486.071,38$ & $135 \%$ \\
$78 \%$ Beca & 17 & $13.306,97$ & $131.994,60$ & $992 \%$ \\
$2 \%$ Recursos Propios 226 & $117.726,92$ & $356.271,17$ & $303 \%$ & $27 \%$ \\
ICETEX & 66 & $56.151,71$ & $260.993,62$ & $465 \%$ & $8 \%$ \\
Ingresos Totales & 835 & $545.575,80$ & $589.591,30$ & $108 \%$ & $100 \%$ \\
\hline
\end{tabular}

Fuente: encuesta aplicada a estudiantes flotantes de pregrado II semestre 2007. U niversidades de Tunja.

Tabla 3. Distribución de ingresos totales clasificados por salarios mínimos legales vigentes. M ensuales

\begin{tabular}{|l|c|c|}
\hline $\begin{array}{l}\text { Ingresos Totales } \\
\text { Por salarios (s. m.I.v) } \\
\text { mínimos vigentes }\end{array}$ & $\begin{array}{c}\text { Número de } \\
\text { Estudiantes }\end{array}$ & $\begin{array}{c}\text { Porcentaje de } \\
\text { Estudiantes }\end{array}$ \\
\hline M enor o igual a 1 s.m.I.v. & 441 & $52 \%$ \\
Entre 1 y 2 s. m. I.v. & 302 & $36 \%$ \\
Entre 2 1 y 3 s.m.I.v. & 77 & $9 \%$ \\
M ayor que 3 s.m.l.v. & 27 & $3 \%$ \\
Total & 847 & $100 \%$ \\
\hline
\end{tabular}

Fuente: encuesta aplicada a estudiantes flotantes de pregrado II semestre 2007. Universidades de Tunja. Observación: 1 salario mínimo legal vigente (s. m.l.v.) equivale a \$433.700,00 en el momento de la encuesta.

1 Un salario mínimo legal vigente (s.m.l.v.) en el momento de la encuesta (año 2007) equivalía a $\$ 433.700,00$ corrientes. 
Estimación y análisis de los gastos e ingresos económicos de la población flotante ...

M aría Isabel Rojas Triana

2.2 Descripción de los gastos de los estudiantes flotantes universitarios

El valor de las matrículas semestrales en las distintas universidades oscila entre uno y diez salarios mínimo, siendo la tarifa media cinco salarios mínimos.

En cuanto a gastos, el promedio total mensual es de $\$ 386.172,30$ corrientes con una desviación de $\$ 325.371,00,10$ que significa que algunos estudiantes tienen gastos cercanos a $\$ 800.000,00$ mientras otros están por debajo de $\$ 60.000,00$ corrientes mensuales aproximadamente. En promedio, los gastos más altos se re- gistran en alimentación, seguido por arriendos, cuyos valores oscilan desde cero, (algunos estudiantes no pagan arriendo, viven en casas de familiares 0 amigos, 0 en residencias estudiantiles), mientras que otros, se estima, pagan desde el valor del arriendo de una habitación, hasta el valor de un apartamento a un precio moderado de $\$ 215.000,00$ corrientes aproximadamente, observando el promedio y la desviación estándar para este rubro. Al mismo nivel, se encuentra el promedio en apoyos académicos (útiles escolares, fotocopias, papelería); y, por último, gastos en transporte (ver Tabla 4).

Tabla 4. Distribución de gastos mensuales (pesos corrientes)

\begin{tabular}{|l|c|c|c|c|c|}
\hline $\begin{array}{c}\text { Estadísticos } \\
\text { descriptivos/ } \\
\text { Destinación del } \\
\text { gasto mensual }\end{array}$ & $\begin{array}{c}\text { Número de } \\
\text { Estudiantes } \\
\text { No pagan }\end{array}$ & $\begin{array}{c}\text { Promedio } \\
\text { (Pesos } \\
\text { corrientes) }\end{array}$ & $\begin{array}{c}\text { Desviación } \\
\text { Típica (Pesos } \\
\text { Corrientes) }\end{array}$ & $\begin{array}{c}\text { Coeficiente } \\
\text { de } \\
\text { Variación }\end{array}$ & $\begin{array}{c}\text { Porcentaje } \\
\text { Estudiantes } \\
\text { No pagan }\end{array}$ \\
\hline $\begin{array}{l}\text { Arriendo } \\
\text { Alimentación }\end{array}$ & 263 & $99.004,57$ & $114.195,12$ & $115 \%$ & $31 \%$ \\
Transporte & 218 & $125.506,49$ & $145.032,79$ & $116 \%$ & $4 \%$ \\
$\begin{array}{l}\text { A poyos académicos } \\
\text { Promedio gastos }\end{array}$ & 5 & $94.594,33$ & $97.577,37$ & $151 \%$ & $26 \%$ \\
mensuales & 847 & $386.172,96$ & $325.370,96$ & $84 \%$ & $100 \%$ \\
\hline
\end{tabular}

Fuente: encuesta aplicada a estudiantes flotantes de pregrado II semestre 2007. U niversidades de Tunja.

Nótese que los coeficientes de variación son altos en todas las variables, indicando que los gastos para estos estudiantes son, en cifras, muy heterogéneos.

La mayor parte de los estudiantes, equi- valente al $51 \%$, se ubican en viviendas de estrato tres, seguidas por un $15.9 \%$ estrato cuatro, mientras que un menor porcentaje $6.8 \% \quad 1.7 \% \quad 1,1 \%$ se ubica en estratos: dos, cinco y uno respectivamente. $23.4 \%$ no registran ${ }^{2}$. (ver gráfica 1).

2 Algunos estudiantes cambian de barrio por diversas razones manifestadas en la encuesta, pero siempre con la finalidad de reducir gastos y ganar bienestar. 
En un día de actividad académica, el $36 \%$ de los estudiantes se trasladan a pie argumentando que para ellos resulta más económico, mientras que el $41 \%$, se transportan en buses y busetas, el $7 \%$ en bicicleta y solo un $2 \%$ y un $1 \%$ Io hacen en auto particular y taxi respectivamente. (Ver gráfica 2).

Grafica 1. Distribución ubicación de vivienda por estratos

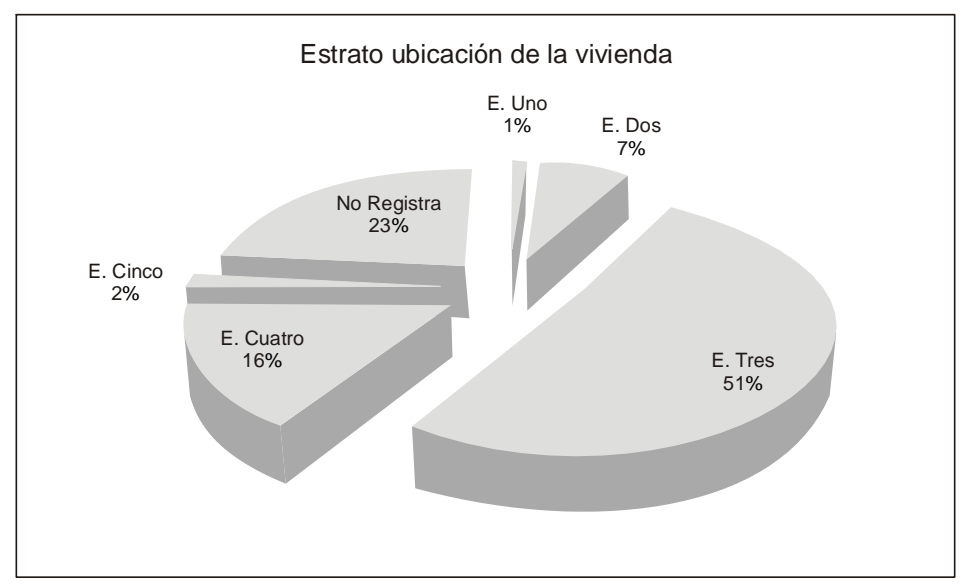

F uente: encuesta aplicada a estudiantes flotantes de pregrado II semestre 2007. U niversidades de Tunja.

Grafica 2. Frecuencia y medio de transporte urbano

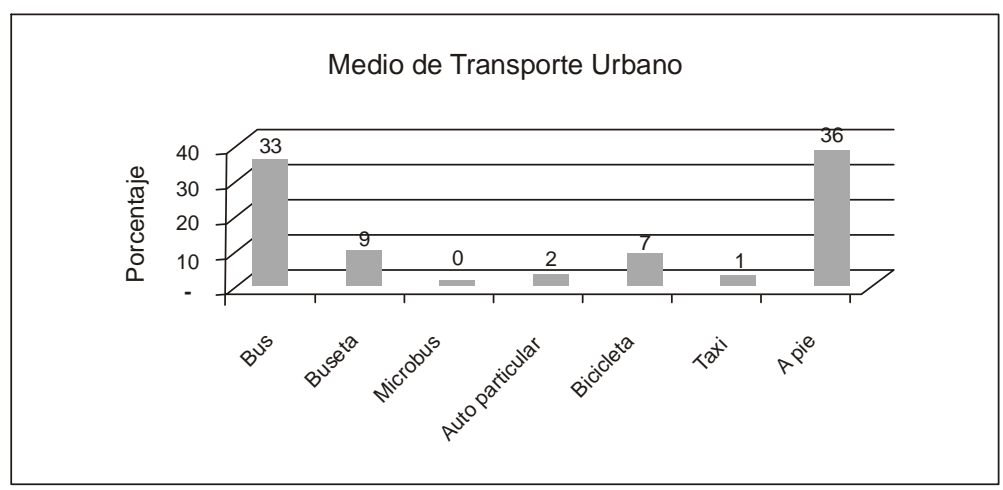

F uente: encuesta aplicada a estudiantes flotantes de pregrado II semestre 2007. U niversidades de Tunja. 
La variación por concepto de transporte se explica por las distancias entre la universidad y la ubicación de sus viviendas, así como por la oferta del servicio de transporte urbano.

\section{Formas funcionales matemáticas que relacionan el ingreso y el gasto}

Para estudiar el comportamiento del gasto e ingreso, se parte de las propiedades que cumplen las funciones de la demanda según la teoría microeconómica del consumidor.

Existen modelos de sistemas completos de ecuaciones de demanda que se han hecho con el fin de analizar el comportamiento de los consumidores, suponiendo que cumplen las hipótesis de la teoría. Entre ellos, se puede mencionar el sistema de Working y Leser, el cual admite datos de corte transversal para su análisis. Permite establecer con mejor precisión información sobre características de la población en vivienda, salud, educación, empleo e ingreso laboral y patrones de gasto, entre otros.

A guiler y Fernández (2002), Ramírez et al. (2004) y Jiménez (2000), citan a autores como Donatos y Mergos (1989), Fulponi (1989), Moschini y Meilke (1989) y Hayes, Wahl y Williams (1990), en los que se pueden apreciar trabajos empíricos aplicados que se ajustan al tema en mención.

Por otro lado, la curva de Engel ha sido muy utilizada para estimar los parámetros de demanda. Desde el punto de vista económico, Varian $\mathrm{Hal}$ (1992) define esta curva como el lugar geométrico de puntos que muestran las diferentes cantidades de un bien adquiridas a diferentes niveles de ingreso-gasto. Esta relación entre el ingreso y el gasto en un bien particular, mantiene los demás factores constantes.

En el análisis de demanda la curva de Engel representa el gasto en un bien como función solo del ingreso. Asimismo, la estimación provee mejores resultados cuando existe cierto grado de homogeneidad, a fin de evitar una excesiva dispersión en los parámetros estimados por Deaton y Muellbauer (1980).

En este estudio se toman como referencia los gastos de un grupo de estudiantes flotantes y se trabaja inicialmente con el supuesto de ingreso conjunto.

EI análisis de corte transversal permite que la información disponible de la encuesta presente una aproximación al comportamiento de las familias, en sus decisiones de gasto a nivel agregado.

En este contexto y dado que los datos son de corte transversal, el presente análisis se centra en la relación de Engel, existente para un grupo específico de bienes y servicios, considerando la condición de 
"ceterisparibus", con el fin de afirmar que esta es una función de demanda derivada de la maximización de una función de utilidad que no requiere información relativa a precios, ya que se asume que estos permanecen constantes.

Inicialmente se parte de los trabajos empíricos de Duncan Thomas (1990; 1997), en donde se utiliza el modelo expuesto por Samuelson (1956), para implementar el sistema de elasticidades de Working y Lesser (Deaton \& M uellbauer, 1980).

La función de utilidad de cada individuo $u_{i}$, se define por:

$$
U=U\left[u_{1}\left[X_{1}\right], \ldots u_{n}\left[X_{n}\right]\right]
$$

Depende del consumo de bienes del total de $\mathrm{N}$ estudiantes; $\mathrm{N}=1, \ldots, \mathrm{n}$ identifica el número total de individuos, donde $X$ corresponde al vector de bienes demandados por los $n$ estudiantes en estudio.

La función de bienestar del estudiante es una función del tipo BergsonSamuelson, que agrega las funciones de utilidad individual de los estudiantes.

El modelo impone como condición necesaria la separabilidad débil entre las funciones de utilidad individual
(Thomas, 1990). La función cumple las características explicadas en Samuelson (1956); la función de utilidad agregada se maximiza, sujeta a la restricción presupuestal de los estudiantes, que se define por:

$$
\mathrm{P} * \mathrm{X}_{\mathrm{j}}=\mathrm{y}
$$

Donde y es el ingreso de los estudiantes, compuesto por los ingresos individuales $\mathrm{y}_{\mathrm{i}}$. Maximizando la función de utilidad sujeta a la restricción presupuestal, se obtiene la función de demanda marshalliana que se puede descomponer para el conjunto de estudiantes como sigue:

$$
X=\sum X_{j}=g\left[p, \sum y_{j}\right]
$$

En forma resumida lo anterior se expresa:

Maximizar la función $U$,

$M$ ax $U(q) ;$ donde $q=\left[u_{1}\left[X_{1}\right]\right.$, $\left.\ldots u_{n}\left[X_{n}\right]\right]$

Sujeto a la restricción $p^{*} q=y=G T^{3}$

$\mathrm{L}$ a función de gasto $g\left[p, \sum y_{j}\right]$, refleja el comportamiento de un estudiante típico. La suma de las demandas es equivalente a la suma del gasto en cada

3 En el análisis de demanda, la curva de Engel representa "el gasto total" (GT) en un bien como función solo del ingreso; puede ser expresada de la siguiente manera: $p_{i}{ }^{*} x_{i}=d_{i}{ }^{*} y$ donde: $p i=$ Precio del bien $i ., x_{i}=$ gasto que se destina al bien $i ., y=$ ingreso total. $d i=$ proporción del ingreso total destinada al gasto en el bien $i$. 
una de ellas y de sus respectivos precios, esto le permite satisfacer la restricción presupuestaria, para obtener que:

$$
\sum_{i} p_{i} \frac{\delta g\left[p, y_{i}\right]}{\delta y}=1
$$

En donde, cambios en precios (p) y gastos (ingresos) $(y=G T)$, generan recomposiciones en la canasta de gasto, que no violan la condición presupuestaria (Deaton \& M uellbauer, 1980); con ese presupuesto los estudiantes comprarán menos del bien que se encuentre afectado por aumento en los precios. La ecuación se conoce en la literatura como la agregación de Engel. Esta permite observar cómo se presenta la participación de cada uno de los grupos de gasto que efectúan los estudiantes, respecto del gasto total -con precios dados para el momento-. Por la restricción de homogenei$\mathrm{dad}^{4}$, con $\mathrm{i}=1, \ldots, \mathrm{n}$, se tiene que:

$$
\sum_{n} p_{n}\left[\frac{\delta g_{i}}{\delta \mathrm{p}_{n}}\right]+\mathrm{y}\left[\frac{\delta g_{i}}{\delta y}\right]=0
$$

La ecuación [6], siguiendo a Deaton y Muellbauer (1980), puede ser expresada en términos del gasto total de los estudiantes como proporciones del gasto, así:

$$
w_{i}=\frac{p_{i} x_{i}}{y}
$$

A sí $w_{i}$ representa la proporción del gasto $y_{i}$ en el bien $j$, respecto del gasto total; donde y representa el gasto total (GT). Se implementa el sistema de Working y Lesser (W-L) en la forma funcional de un modelo de regresión lineal simple (nivel- log) para cada uno de los bienes, como sigue:

$$
\mathrm{w}_{\mathrm{ij}}=\alpha_{\mathrm{i}}+\beta_{\mathrm{i}} * \ln (\mathrm{GT})_{\mathrm{j}}+\varepsilon_{\mathrm{ij}}
$$

donde:

$w_{i j}$ es la proporción de gasto per cápita que el j-ésimo individuo realiza en el iésimo bien.

$\alpha_{i}=$ proporción fija promedio de gasto en el bien i.

$\boldsymbol{\beta}_{\mathrm{i}}=$ es el cambio en la i-ésima proporción de gasto respecto al cambio en el gasto real.

$G T_{j}=$ gasto total per cápita del j-ésimo individuo.

$\varepsilon_{\mathrm{ij}}=$ término de error, $\varepsilon_{\mathrm{ij}} \sim \mathrm{N}\left(0, \sigma^{2}\right)$

U na característica fundamental de esta formulación es que si es aplicada a todos los bienes del presupuesto estudiantil, predice que la suma de todas las proporciones del presupuesto suman la unidad, cumpliendo así las

\footnotetext{
Si se multiplica tanto $p$ como GT por una constante $I$, (con $I>0$ ), I indica una cantidad constante ampliada o aumentada. La demanda permanece invariable, es decir: $\operatorname{Sig}\left(I^{*} \mathrm{p}, I^{*} \mathrm{GT}\right)=\mathrm{g}(\mathrm{p}, \mathrm{GT})$. Entonces la función es homogénea de grado cero en precios y gasto total. No hay ilusión monetaria, lo importante son los precios relativos.
} 
condiciones de "adding-up" de la demanda, agregación de Engel y de Cournot.

El sistema de W - $L$ es empleado de forma frecuente en análisis de corte transversal (Muñoz, 1990; 1998) que permite hacer estimaciones de la curva de Engel (Deaton \& M uellbauer, 1980). Los parámetros estimados son $\alpha_{i}$ y $\beta$ i Como se está trabajando con precios constantes, la propiedad de agotamiento del gasto, que es un requerimiento implica que:

$$
\sum_{n} w_{i}=1
$$

lo que requiere que:

$$
\sum_{n} \alpha_{i}=1 \quad \text { y } \quad \sum_{n} \beta_{i}=0
$$

Si bien es cierto que los coeficientes $\beta$ i no tienen dimensión (es decir, son parámetros), por lo tanto, pueden ser comparados en el tiempo y entre países; a menudo es conveniente convertir los $\boldsymbol{\beta}$, en elasticidades de gasto total. Lo cual se puede hacer de la siguiente manera:

Partiendo de la ecuación [8] y por definición de la elasticidad gasto

$$
e_{i}=\frac{\delta q_{i}}{\delta G T} * \frac{G T}{q_{i}}
$$

Se tiene que:

$$
\hat{\mathrm{e}}_{i}=\bar{w}_{i} *\left(\frac{1}{\bar{W}_{i}}\right)+\beta_{i} *\left(\frac{1}{\bar{w}_{i}}\right)
$$

Donde,

$$
\hat{\mathrm{e}}_{i}=1+\frac{\beta_{i}}{\bar{w}_{i}}
$$

es la estimación de elasticidad gasto del bien i. señalan la relación entre el cambio en el gasto del grupo de estudiantes analizado, respecto al cambio del gasto total (GT).

Es necesario notar que estas elasticidades no son constantes mientras $w_{i} y$ GT varíen. Por lo tanto, normalmente se calculan con respecto a la media muestral de $\mathrm{w}_{\mathrm{i}}$. Notada como $\overline{\mathrm{w}}_{\mathrm{i}}$.

La fórmula $\left(e_{i}\right)$ implica que si la elasticidad del gasto es distinta de 1, el coeficiente $\beta_{i}$ determina el valor final de la elasticidad. Si $\beta_{i}$ es positivo, se trataría de un bien suntuario; si es negativo, se tendría un bien necesario.

La elasticidad de los bienes clasificados como necesarios se encuentra en el intervalo $0<e<1$, los bienes de lujo presentan una e $>1$, en los bienes inferiores la e $<0$. Los bienes con e $=1$ presentan curvas de Engel que son líneas rectas que parten del origen. Los $\hat{a}_{i}$ muestran la proporción que gastarían los estudiantes en el grupo de bienes, si aumentara o no su capacidad de gasto total (GT). Si $\beta_{i}>$ 0 expresa bienes de lujo, y $\beta_{i}<0$ bienes necesarios 0 inferiores.

L as estimaciones utilizan los parámetros de forma reducida para 
obtener los de la forma estructural. La forma reducida del sistema de ecuaciones lineales, en su representación será:

WALIMENT $=\alpha_{1}+\beta_{1} * \ln (G T)+\varepsilon_{1}[14]$ WARRIEND $=\alpha_{2}+\beta_{2} * \ln (G T)+\varepsilon_{2}[15]$ WTRANSPO $=\alpha_{3}+\beta_{3} * \ln (G T)+\varepsilon_{3}[16]$ WAPACADE $=\alpha_{4}+\beta_{4} * \ln (G T)+\varepsilon_{4}[17]$

Donde WALIMENT es la proporción del gasto per cápita en alimentación, WARRIEND es la proporción del gasto per cápita en arriendos, WTRANSPO es la proporción del gasto per cápita en transporte y para WAPACA es la proporción del gasto per cápita en apoyos académicos.

La propiedad pertinente al agotamiento del gasto se cumple con las dos condiciones anteriores y está garantizada si cada ecuación del sistema se estima con M ínimos Cuadrados Ordinarios (MCO).

El término constante o intercepto $\alpha_{i}$ representa la proporción fija promedio de gasto que se destina al bien i cuando el logaritmo del gasto per cápita (GT) permanece igual a 1. El coeficiente $\beta_{i}$ representa el cambio en la iésima proporción de gasto respecto al cambio en el ingreso (gasto) real, su- poniendo todo lo demás constante. Wooldridge (2001, p. 45) expresa el cambio en cada proporción como el porcentaje:

$$
\Delta w_{i}=\left(\beta_{i} / 100\right) \% \Delta G T
$$

Para todos aquellos bienes cuyo coeficiente $\beta>0$, se espera que un incremento del gasto per capita conduzca a un aumento del gasto en el bien, el cual se considera como un bien de lujo; por el contrario, si $\beta_{1}<0$, la proporción de gasto disminuiría con relación al gasto per cápita, en este caso se trataría de un bien necesario.

\section{Resultados, análisis y discusión}

La estimación del sistema de ecuaciones se hizo mediante el método de mínimos cuadrados ordinarios, para las ecuaciones [14], [15] y [16] y por mínimos cuadrados ponderados para la ecuación [17], dado que su distribución no se normalizó completamente y tenía problemas de heterocedasticidad, I os datos son procesados con el programa SPSS (Statistical Packagefor Social Sciences) y los resultados en forma resumida se muestran en la tabla 5 junto con las ecuaciones estimadas, como sigue: 
WALIMENT $=$

$0.65-0.02 * \ln (12.61)+\varepsilon_{1}$

WARRIEND $=$

$-0.62+0.07 * \ln (12.62)+\varepsilon_{2}$

[20]

WTRANSPO $=$

$0.24-0.01 * \ln (12.69)+\varepsilon_{3}$

WAPACADE $=$

$0.73-0.04 * \ln (12.62)+\varepsilon_{4}$

Los resultados se centran en el impacto que tiene el gasto total de los estudiantes flotantes sobre cada una de las destinaciones del mismo (variables dependientes), tales como: alimentación, arriendo, transporte y apoyos académicos, medidos a través de la significancia estadística de los coeficientes; sin tener en cuenta la bondad de ajuste medida por el R-cuadrado. Generalmente, el bajo valor del R-cuadrado refleja la enorme dispersión de los datos individuales en muestras de tipo microeconómico, tan amplias como la que se analiza en el presente estudio.

Todos los valores del estadístico de Durbin Watson (columna 9) se encuentran entre 1.5 y 2.5 , luego puede asumirse que los residuos son independientes. LoS gráficos P-P normal de regresión de los residuos mostraron en todas y cada una de las variables dependientes Wi que los residuos 
se distribuyen normalmente, lo cual se identifica cuando la probabilidad acumulada observada es aproximadamente igual a la probabilidad acumulada esperada (trazando una línea de $45^{\circ}$ sobre el plano) e igualmente los gráficos de dispersión de la varianza indicaron homocedasticidad identificando su dispersión dentro de una banda horizontal. Los gráficos de dispersión para la variable dependiente proporción apoyos académicos, no se generaron puesto que la estimación se llevó a cabo por mínimos cuadrados ponderados (estos resultados no se muestran en el artículo, pero se pueden consultar en el trabajo realizado por la autora Rojas Triana (2009).

Lo anterior indica que no hay razones para pensar que los residuos incumplan los supuestos de normalidad, independencia y varianza constante. A demás se puede observar que la hipótesis del modelo es válida a un nivel de significancia del $5 \%$, dado el p-valor de significancia igual a 0 , cantidad menor al nivel establecido, en todos los casos.

Se puede observar que la hipótesis nula $\beta_{i}=0$ es rechazada para todas $y$ cada una de las regresiones estimadas a un nivel de significancia de 0.05 , dados los valores p- de significancia (columna 5) menores a 0.05 (punto de comparación). Luego concluimos que las ecuaciones de demanda estimadas [19], [20], [21] y [22] son validadas para su predicción.
Se puede evidenciar, por el valor de los coeficientes de Beta_i, que la proporción de gasto en arriendo (0.07) capta el mayor impacto de los cambios en el gasto total. Ello podría hacer pensar que los estudiantes al aumentar su gasto total reasignan una mayor proporción de gasto en dirección a los servicios de arriendo reduciendo gastos en alimentación $(-0,02)$, transporte $(-0,01)$ y apoyos académicos $(-0,04)$ (útiles escolares, fotocopias...). A unque estos últimos, para los estudiantes en todos los casos son considerados como bienes necesarios (aquellos que presentan elasticidad entre 0 y 1 ), reflejados en las elasticidades de $0.93,0.96$, y 0,85 respectivamente. (V er columna 10). Es importante anotar que si la alimentación, transporte y apoyos académicos son para ellos bienes necesarios, es de esperar que la elasticidad 1.28 en vivienda se interprete para ellos como bien de lujo, como resultó ser el servicio de arriendo en vivienda.

En el caso de que llegaran más estudiantes flotantes, en cantidad superior a los existentes, los resultados muestran que la proporción del gasto en arriendo aumentaría, siendo estadísticamente significativo y consistente con el signo esperado, mientras que el destinado a alimentación, transporte y apoyos académicos, el que sus coeficientes betas hayan sido negativos y su elasticidad menor que uno, no quiere decir que no se va aumentar el gasto en esos servicios, quiere decir que la proporción no 
aumentará al incrementarse el gasto total, pero si el nivel de gasto es proporcional al aumento de la población, dado que las elasticidades de gasto son cercanas a 1.

En este orden, la proporción del gasto en transporte y alimentos aumentaría casi en igual proporción al gasto total y donde menos aumentaría sería en apoyos académicos, siendo todos ellos también estadísticamente significativos.

De otro lado, si comparáramos estos resultados con el estudio hecho por M uñoz (1999) sobre el comportamiento del gasto en el consumo de algunos bienes y servicios en Tunja, basado en la encuesta de hogares, encontramos que el comportamiento de la población flotante estudiantil con relación a la población tunjana es en algunos casos muy diferente. La elas- ticidad del gasto en alimentos para Tunja fue de 0,79993, inferior en 14 puntos a la población flotante, en transporte 1,24408 superior en 28 puntos a la población flotante y en vivienda 0,72698 inferior en 56 puntos a la población flotante comparado con los registros $0.93,0,96$ y 1,28 respectivamente, de la población estudiantil flotante universitaria (ver tabla 6 Sistema de Working y Leser, columna ELAS_GTO y la columna 9 de la tabla 5). Esta marcada diferencia, sobre todo en la vivienda, se debe a que esta población estudiantil probablemente destina la mayor parte de sus ingresos solo para consumo y no para inversión o ahorro, como lo haría parte de la población local. En particular la tenencia de vivienda es muy diferente para las dos poblaciones o puede ser razonable por la condición de la tenencia de la vivienda comparada en las dos poblaciones. 
Estimación y análisis de los gastos e ingresos económicos de la población flotante... M aría Isabel Rojas Triana

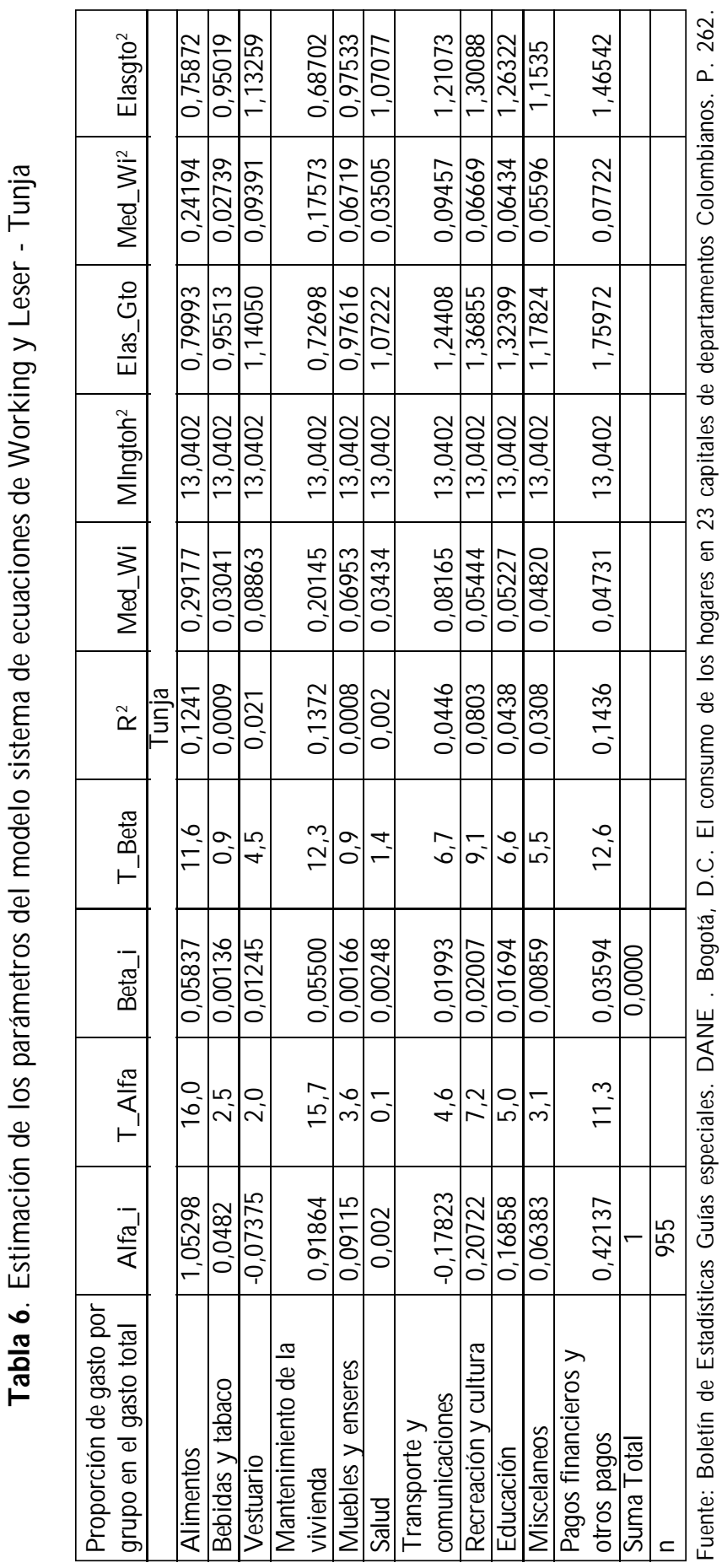




\section{Conclusiones}

Teniendo en cuenta que, como se encontró en la presente investigación, la participación de la población flotante es alta con respecto a la población matriculada, el impacto económico de la población flotante estudiantil de pregrado es positivo en la medida que incrementa la demanda por el consumo de bienes y servicios, en particular el alquiler de vivienda, actividad que aumentaría los ingresos de las familias tunjanas y en consecuencia los de la ciudad.

Al caracterizar la información se puede evidenciar con las distintas elasticidades la relación que existe entre la proporción de gasto total de los estudiantes flotantes universitarios sobre algunos bienes relacionados con el consumo, como lo son en alimentación, transporte, apoyos académicos y arriendo en vivienda, revelando que el mayor impacto de los cambios en el gasto total se representa en la vivienda e interpretándose este bien como un bien de lujo y considerando la alimentación, transporte y apoyos académicos (útiles escolares, fotocopias...) como bienes necesarios.

Si se incrementara el número de estudiantes flotantes en la ciudad o si aumentaran los ingresos totales de los estudiantes se reasignará una mayor proporción de gasto en dirección a los servicios de arriendo, reduciendo en menor nivel al encontrado la proporción de gastos en alimentación, transporte y apoyos académicos (útiles escolares, fotocopias...) aunque estos últimos bienes considerados como bienes necesarios son importantes para priorizar atención y acción en aspectos relacionados con el consumo, dado que los requerimientos de estos servicios son básicos y lo encontrado es que pareciera que cada vez más disminuyera, si sus gastos aumentaran causando menos consumo local.

Por otra parte este comportamiento se refleja en la descripción de la distribución de los ingresos y de los gastos, si se tiene en cuenta que buena parte de los estudiantes, correspondiente a más de la mitad de ellos, revelaron tener ingresos menores a 1 salario mínimo, y que aproximadamente la mitad de ellos comparten habitación, aunado a la tendencia a cambiar de vivienda relacionada con la ubicación y estrato de las mismas. La movilidad y la forma de transportarse en días de actividad académica, siempre reduciendo distancias de sus residencias a la universidad y en consecuencia disminuyendo gastos por concepto de transporte y vivienda. Los gastos por concepto de apoyos académicos son inevitables y por lo tanto reflejan poca variación de asignación de gastos proporcional con respecto al gasto total en cualquier caso.

A demás una mayor provisión de los anteriores servicios proporciona a los estudiantes flotantes una vida mejor en 
el entendido de que alimentación, transporte y apoyos académicos son los el ementos fundamentales para el desarrollo integral y futuro del mercado laboral, con mejores oportunidades.

Los valores estimados a partir de los datos de las encuestas también sirven como parámetros de economías para comparar si estos están sobre o por debajo de un valor crítico, como se ve la marcada diferencia del comportamiento en el consumo de la población local y la población flotante estudiantil sobre todo en vivienda, estos resultados pueden ser considerados relevantes para la realidad de tal forma que se obtienen implicaciones de política en población flotante.
Finalmente, se concluye que si bien el modelo propuesto, proporciona parámetros que miden el impacto del gasto (ingreso) sobre el consumo de determinados bienes, no incluye factores demográficos ni socioeconómicos que podrían modificar en cierta medida los coeficientes estimados inicialmente. Es posible introducir variables "dummies" para captar el impacto de las variables propuestas. Sin embargo, se puede ir más lejos en la especificación de modelos que contemplen variaciones más desagregadas de variables tales como edad, sexo, estado civil entre otras, los cuales se dejan para posteriores estudios.

\section{R eferencias bibliográficas}

1. A guilera, A. \& Fernández J. (2002). Pobreza y tamaño de la familia: economías de tamaño y escalas de equivalencia demográficas para el Paraguay. En Libros en Google Play.

2. Deaton, A. \& Muellbauer, J. (1980) E conomics and consumer behavior, $\mathrm{N}$ ew Y ork, C ambridge U niversity Press.

3. Dureau, F,. (2001). El proceso migratorio y sus consecuencias sobre el poblamiento de las ciudades petroleras: realidades y representaciones colectivas en el caso de las ciudades de casanare, colombia. Revista Electrónica de Geografía y Ciencias Sociales.
Migración y cambio social. U niversidad de Barcelona.

4. Jiménez, Z. (2000). Una aplicación empírica de la curva de Engel. Bolivia. enwww. udape.gov. bo/analisis Economico/analisis/vol12/art04. pdf

5. Leiwen, J. ( 2006). Living Conditions of the floating population in urban china. Watson institute for international studies. Brown U niversity. Providences USA, 21 (5), p. 719-744.

6. M uñoz, M. (1999). El consumo de los hogares en 23 capitales de departamentos Colombianos. Boletín de 
Estadísticas Guías especiales. Bogotá: DANE. Bogotá.

7. Ramírez, M . et al. (2004). Respuesta de la oferta y la demanda Agrícola en el marco de un TLC con Estados Unidos. M inisterio de A gricultura y Desarrollo Rural. Observatorio A grocadenas Colombia. Bogotá.

8. Rojas, M. (2009) Impacto socioeconómico de la población flotante estudiantil universitaria de pregrado en Tunja. Tesis de Grado M aestría en Economía. Convenio UPTC. Universidad Nacional de Colombia. Bogotá.

9. Varian, H. (1992) Microeconomic Analysis. Third edition. Norton international student.

10. W ooldridge, J. (s.f.). Introducción a la Econometría. México y América Central: Thompson Learning. 